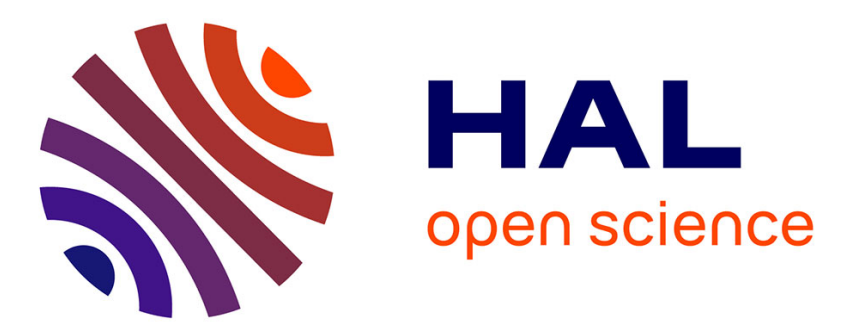

\title{
Anatomical study of the medial plantar proper digital nerve using ultrasound
}

Thomas Le Corroller, Elodie Santiago, Arnaud Deniel, Anne Causeret, Pierre Champsaur, Raphaël Guillin

\section{- To cite this version:}

Thomas Le Corroller, Elodie Santiago, Arnaud Deniel, Anne Causeret, Pierre Champsaur, et al.. Anatomical study of the medial plantar proper digital nerve using ultrasound. European Radiology, 2019, 29 (1), pp.40-45. 10.1007/s00330-018-5536-6 . hal-02528745

\section{HAL Id: hal-02528745 \\ https://hal.science/hal-02528745}

Submitted on 14 Apr 2020

HAL is a multi-disciplinary open access archive for the deposit and dissemination of scientific research documents, whether they are published or not. The documents may come from teaching and research institutions in France or abroad, or from public or private research centers.
L'archive ouverte pluridisciplinaire HAL, est destinée au dépôt et à la diffusion de documents scientifiques de niveau recherche, publiés ou non, émanant des établissements d'enseignement et de recherche français ou étrangers, des laboratoires publics ou privés. 


\title{
Anatomical study of the medial plantar proper digital nerve using ultrasound
}

\author{
Thomas Le Corroller $^{1,2} \cdot$ Elodie Santiago $^{1,2} \cdot$ Arnaud Deniel $^{3} \cdot$ Anne Causeret $^{3} \cdot$ Pierre Champsaur $^{1,2} \cdot$ Raphaël $^{2}$ \\ Guillin $^{3}$
}

\begin{abstract}
Purpose To determine whether ultrasound allows precise assessment of the course and relations of the medial plantar proper digital nerve (MPPDN).

Materials and methods This work was initially undertaken in six cadaveric specimens and followed by a high-resolution ultrasound study in 17 healthy adult volunteers (34 nerves) by two musculoskeletal radiologists in consensus. Location and course of the MPPDN and its relationship to adjacent anatomical structures were analysed.

Results The MPPDN was consistently identified by ultrasound along its entire course. Mean cross-sectional area of the nerve was $0.8 \mathrm{~mm}^{2}$ (range 0.4-1.4). The MPPDN after it branches from the medial plantar nerve was located a mean of $22 \mathrm{~mm}$ (range 1927) lateral to the medial border of the medial cuneiform. More distally, at the level of the first metatarsophalangeal joint, mean direct distances between the nerve and the first metatarsal head and the medial hallux sesamoid were respectively $3 \mathrm{~mm}$ (range 18) and $4 \mathrm{~mm}$ (range 2-9).

Conclusion The MPPDN can be depicted by ultrasonography. Useful bony landmarks for its detection could be defined. Precise mapping of its anatomical course may have important clinical applications.

Key Points

- The medial plantar proper digital nerve (MPPDN) rises from the medial plantar nerve to the medial side of the hallux.

- Because of its particularly long course and superficial position, the MPPDN may be subject to trauma, resulting in a condition known as Joplin's neuroma.

- The MPPDN can be clearly depicted by ultrasound along its entire course. Precise mapping of its anatomical course may have important clinical applications.
\end{abstract}

\section{Introduction}

The medial plantar proper digital nerve (MPPDN) is a terminal branch nerve arising from the medial plantar nerve, which, in turn, is the larger and terminal branch of the tibial nerve [1, 2]. The MPPDN originates from the medial side of the medial plantar nerve below the abductor hallucis muscle (Fig. 1). In its course in the medial compartment, the nerve is located between the flexor hallucis brevis medially and flexor digitorum brevis laterally, and gives off a muscular branch to the flexor hallucis brevis [3]. The nerve pierces the plantar fascia posterior to the tarsometatarsal joint and courses distally through subcutaneous tissues (Fig. 1). It supplies innervation to the medial plantar aspect of the first metatarsophalangeal joint (MTPJ), hallux and tip of the toe [3-5]. 
Fig. 1 (a) Plantar schematic anatomical view of a right foot illustrating the medial plantar proper digital nerve (arrowheads) originating from the medial side of the medial plantar nerve (long arrow) below the abductor hallucis (AH) muscle. (b-d) Coronal T1-weighted SE MR images of a right foot exhibiting the medial plantar proper digital nerve (MPPDN) (arrowhead) course distally through subcutaneous tissues

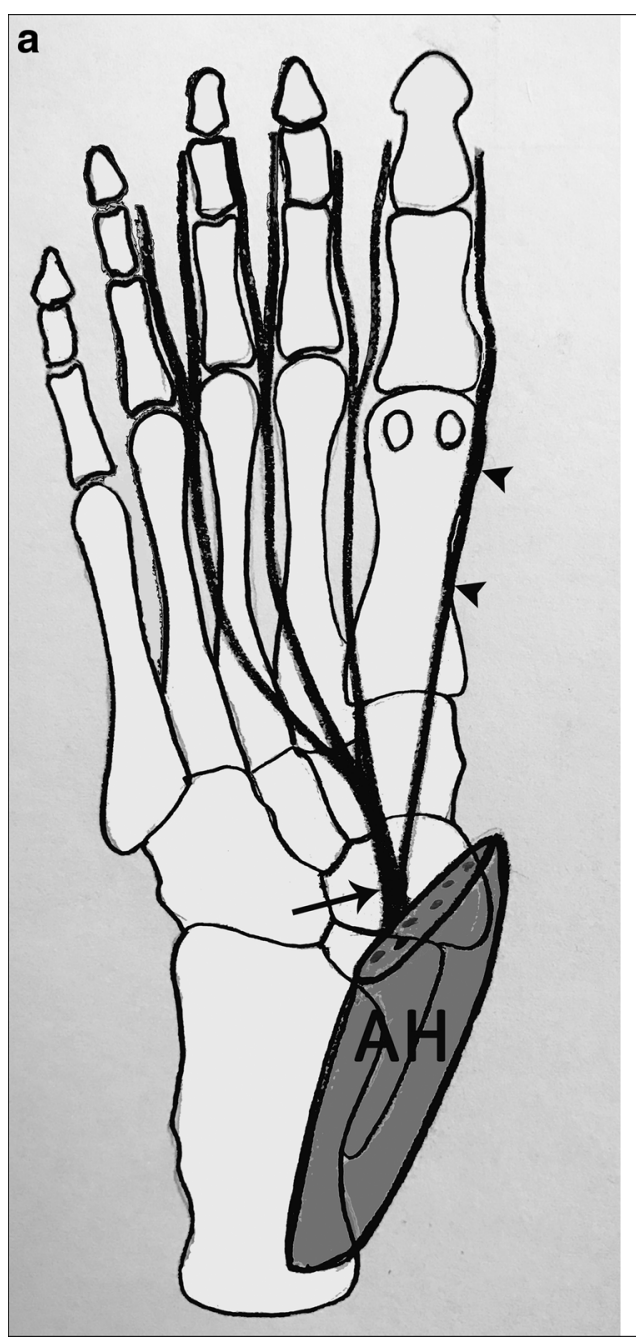

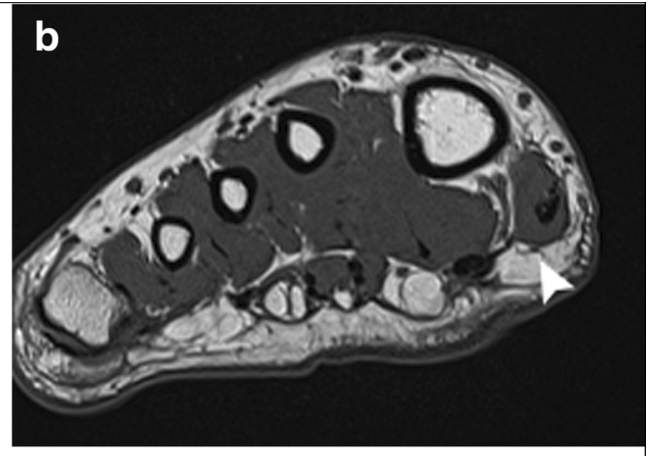
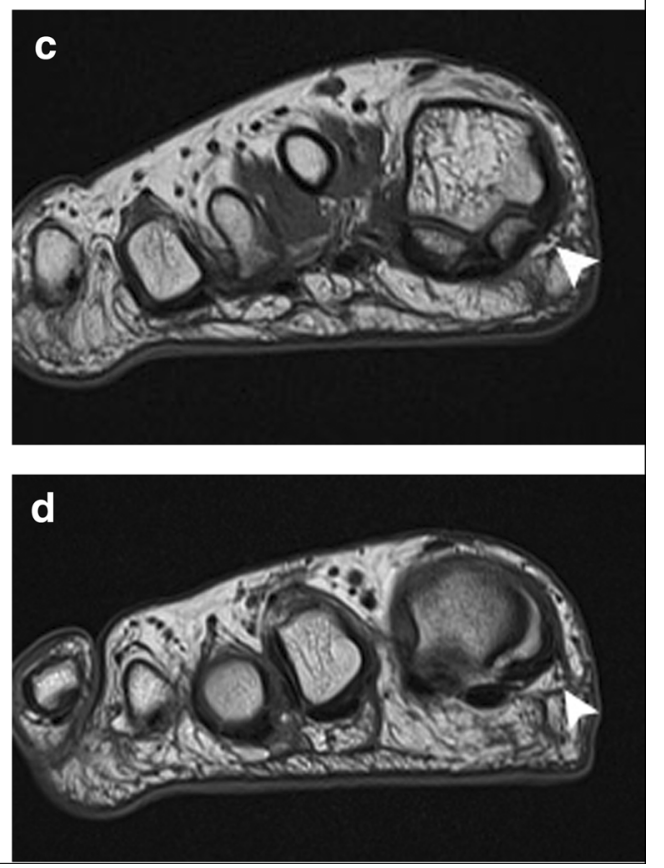

Because of its particularly long course and superficial position, the MPPDN may be subject to trauma, which results in a condition known as Joplin's neuroma [6]. Repetitive microtrauma to the nerve during gait may occur in patients who are anatomically predisposed by the presence of a first metatarsal prominent bony ridge. Another cause of Joplin's neuroma is biomechanic derangement with a plantar-flexed first ray and forefoot valgus. Overpronation during the propulsive period of gait may cause abnormal shearing forces, which can result in progressive perineural fibrosis of the nerve [3]. In the same way, sporting activities that require repetitive pivoting, impact and motion at the first MTPJ, such as running, soccer, basketball, snow skiing and ballet dancing, may cause the nerve to become enlarged and painful [3]. Clinically, patients present with numbness, dysaesthesia and pain of the medial plantar aspect of the great toe. Tinel's sign can often be elicited on percussion on the medial plantar aspect of the first MTPJ [3]. Differential diagnosis typically includes medial hallux sesamoid pathology, turf toe, arthritis and bursitis.

Conditions that affect the peripheral nerves are generally evaluated through a combination of history, clinical examination and electrodiagnostic studies. However, because electrodiagnostic studies neither allow visualisation of intrinsic abnormalities of peripheral nerves nor provide information about their relationship to adjacent anatomical structures, high-resolution ultrasound (US) has become an attractive complement for assessing the peripheral nervous system [7, 8]. First, US is known to offer certain advantages over other imaging modalities: it is a readily available, non-invasive and cost-effective method. Then, in the setting of a superficial neuropathy, US provides higher spatial resolution than magnetic resonance (MR) imaging and characterizes tissue movement in real time, making it an ideal imaging technique.

Still, to the best of our knowledge, no description of the MPPDN by means of sonography has been reported. Because precise mapping of the course of this nerve may have significant clinical implications, such as avoiding injury to it during bunion surgery, diagnosing iatrogenic injuries or guiding an elective neural blockade, the aim of our study was to determine whether US allows precise assessment of the MPPDN and its relationship to adjacent anatomical structures. 


\section{Materials and methods}

\section{Anatomical study in cadavers}

In compliance with institutional safety and ethics regulations, this study was initially undertaken on six formalin-embalmed cadaveric lower limbs amputated at the distal leg level (one male and two female, mean age at death 89 years) to preliminarily assess the US depiction of the MPPDN and gain a better understanding of its anatomical course. None of the cadavers showed prior evidence of external foot injuries or surgical procedures on the ankle and foot region. The specimens were placed in lateral rotation and were examined using an iU22 (Philips Medical Systems) and a 17.5-MHz linear probe. A staff radiologist with 12 years of experience and a fellow in musculoskeletal radiology performed all the examinations in consensus. The visibility of the MPPDN was scored according to a four-level scale: 0 - not visible; 1 -identified with difficulty; 2 - clearly identified without internal nerve structure visibility; and 3-clearly identified with internal nerve structure visibility [9]. The cross-sectional area and course of the MPPDN were recorded. Bony landmarks for nerve detection were defined. First, the distance between the MPPDN after it branches from the medial plantar nerve and the medial border of the medial cuneiform was measured using US (Fig. 2a). Second, at the level of the first MTPJ,
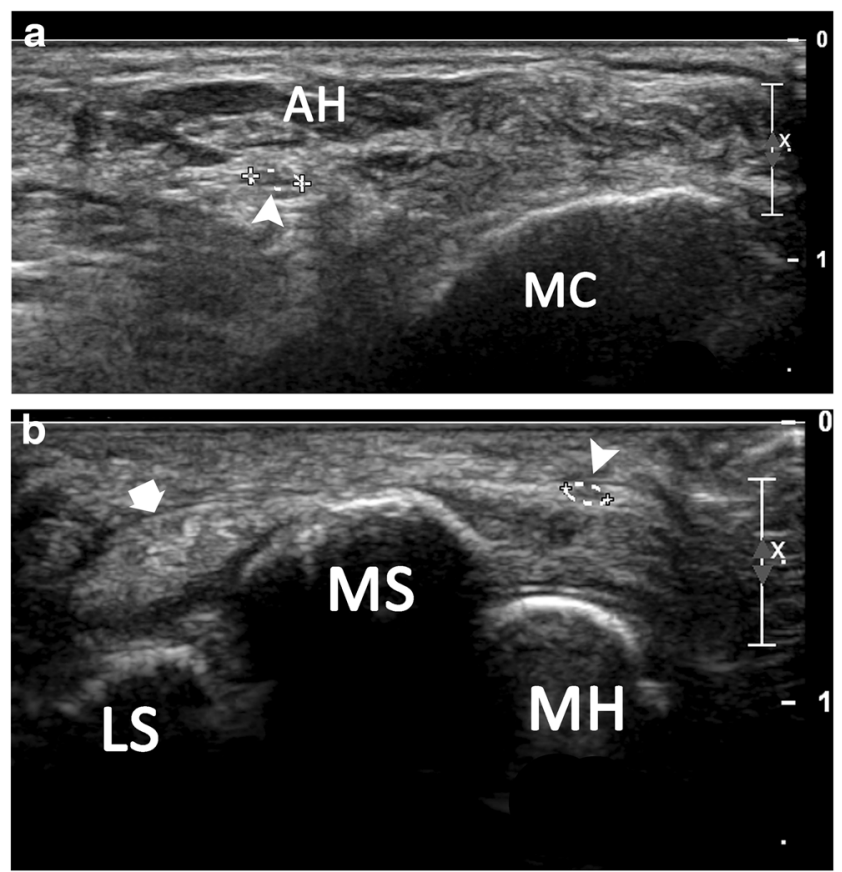

Fig. 2 Axial US images obtained in a cadaveric specimen showing a hypoechogenic ovoid structure (arrowhead) along the theoretical course of the medial plantar proper digital nerve (MPPDN) at the level of the medial cuneiform below the abductor hallucis (AH) muscle (a), and at the level of the medial and lateral sesamoids (MS and LS) of the hallux medially to the flexor hallucis longus tendon (thick arrow) (b) the direct distances between the nerve and the first metatarsal head and the medial hallux sesamoid were noted (Fig. 2b). Then, China ink was injected under ultrasound guidance in contact with the presumed MPPDN at its point of branching from the medial plantar nerve and at the level of the first MTPJ. Finally, a cadaver dissection was performed to validate the US data. A skin and subcutaneous flap was reflected from the tibial malleolus to the medial aspect of the hallux. The medial plantar nerve was identified distal to the tarsal tunnel. The course of the MPPDN was analysed, and the three previously defined distances were measured again.

\section{Ultrasound study in volunteers}

After giving their informed consent, 17 healthy adult volunteers, seven males and ten females (mean age 32 years, age range, 24-43 years) with a mean body mass index (BMI) of 21.5 (range 19.5-25) participated in the study. The volunteers presented no traumatic or surgical history, and no symptoms referable to the peripheral nervous system. A second team of two new operators, another staff radiologist with 12 years of experience and a fellow in musculoskeletal radiology performed all the examinations in consensus. Patients were examined in the supine position with lateral rotation of the foot using an iU22 (Philips Medical Systems) and a 17.5-MHz linear probe. Initially, the probe was placed in a transverse direction at the midfoot level. At this location, the medial plantar nerve was identified below the abductor hallucis muscle and followed distally. The MPPDN could be seen as a distinctive nerve that was separated from the medial plantar nerve. Power Doppler was used in all examinations to differentiate the nerve from the adjacent vessels. The visibility, cross-sectional area and course of the MPPDN and its relations to the surrounding structures were then analysed in realtime while imaging. The previously defined distances between the nerve and the medial border of the medial cuneiform, and, more distally, at the level of the first MTPJ, between the nerve and the first metatarsal head and the medial hallux sesamoid were recorded bilaterally.

\section{Results}

The anatomical data obtained from the cadavers are shown in Table 1 . In every cadaveric specimen, the axial US images identified a hypoechogenic ovoid structure along the theoretical course of the MPPDN. The China ink could be injected in the vicinity of the nerve under US guidance in every case at its point of branching from the medial plantar nerve and at the level of the first MTPJ. The mean visibility score of the MPPDN was 2 (range 1-3). The mean cross-sectional area of the nerve was $1.1 \mathrm{~mm}^{2}$ (range 0.9-1.5). The MPPDN after it branches from the medial plantar nerve was located a mean 
Table 1

Anatomical results of six cadaveric specimens

\begin{tabular}{lll}
\hline & US results & Anatomical dissection \\
\hline Nerve visibility score $(0-3)$ & $2(1-3)$ & N/A \\
Cross-sectional area (mm2) & $1.1(0.9-1.5)$ & N/A \\
D medial cuneiform (mm) & $20(18-22)$ & $21(19-22)$ \\
D first metatarsal head (mm) & $8(4-13)$ & $7(4-11)$ \\
D medial hallux sesamoid (mm) & $3(1-5)$ & $4(2-5)$ \\
\hline
\end{tabular}

Data shown include: the medial plantar proper digital nerve (MPPDN) visibility score $(0$ - not visible; 1 -identified with difficulty; 2 - clearly identified without internal nerve structure visibility; 3 - clearly identified with internal nerve structure visibility); the MPPDN cross-sectional area; and the mean distances, respectively, between the MPPDN after it branches from the medial plantar nerve and the medial border of the medial cuneiform, and at the level of the first metatarsophalangeal joint, between the nerve and the first metatarsal head, and between the nerve and the medial hallux sesamoid

of $20 \mathrm{~mm}$ (range 18-22) lateral to the medial border of the medial cuneiform. More distally, at the level of the first MTPJ, mean direct distances between the nerve and the first metatarsal head and the medial hallux sesamoid were respectively 8 $\mathrm{mm}$ (range 4-13) and $3 \mathrm{~mm}$ (range 1-5).

Subsequent cadaver dissection confirmed that the injected structure visualized on US in fact represented the MPPDN (Fig. 3a). The same mean distances measured anatomically were $21 \mathrm{~mm}, 7 \mathrm{~mm}$ and $4 \mathrm{~mm}$, respectively. Interestingly, in four cases, the MPPDN demonstrated a slight enlargement at the level of the medial hallux sesamoid (Fig. 3b).

The US data obtained in 17 volunteers ( 34 nerves) are shown in Table 2. The mean visibility score of the MPPDN was 2 , which indicates that the nerve was consistently clearly identified without internal nerve structure visibility. The mean cross-sectional area of the nerve was $0.8 \mathrm{~mm}^{2}$ (range 0.4-1.5). The mean distance measured between the nerve after it branches from the medial plantar nerve and the medial border of the medial cuneiform was $22 \mathrm{~mm}$ (range 19-27). More distally, at the level of the first MTPJ, mean direct distances between the nerve and the first metatarsal head and the medial hallux sesamoid were respectively $3 \mathrm{~mm}$ (range 1-8) and 4 $\mathrm{mm}$ (range 2-9). The MPPDN could be identified along the majority of its course, from its point of branching from the medial plantar nerve distal to the tarsal tunnel to the medial side of the hallux (Fig. 3). Expectedly, the plantar and dorsal terminal branches of the MPPDN that supply sensation to the tip of the hallux were difficult to assess, given their small size and the adjacent hyperechoic subcutaneous fat.

\section{Discussion}

The MPPDN is a terminal branch nerve arising from the medial plantar nerve. The medial plantar nerve then divides at the
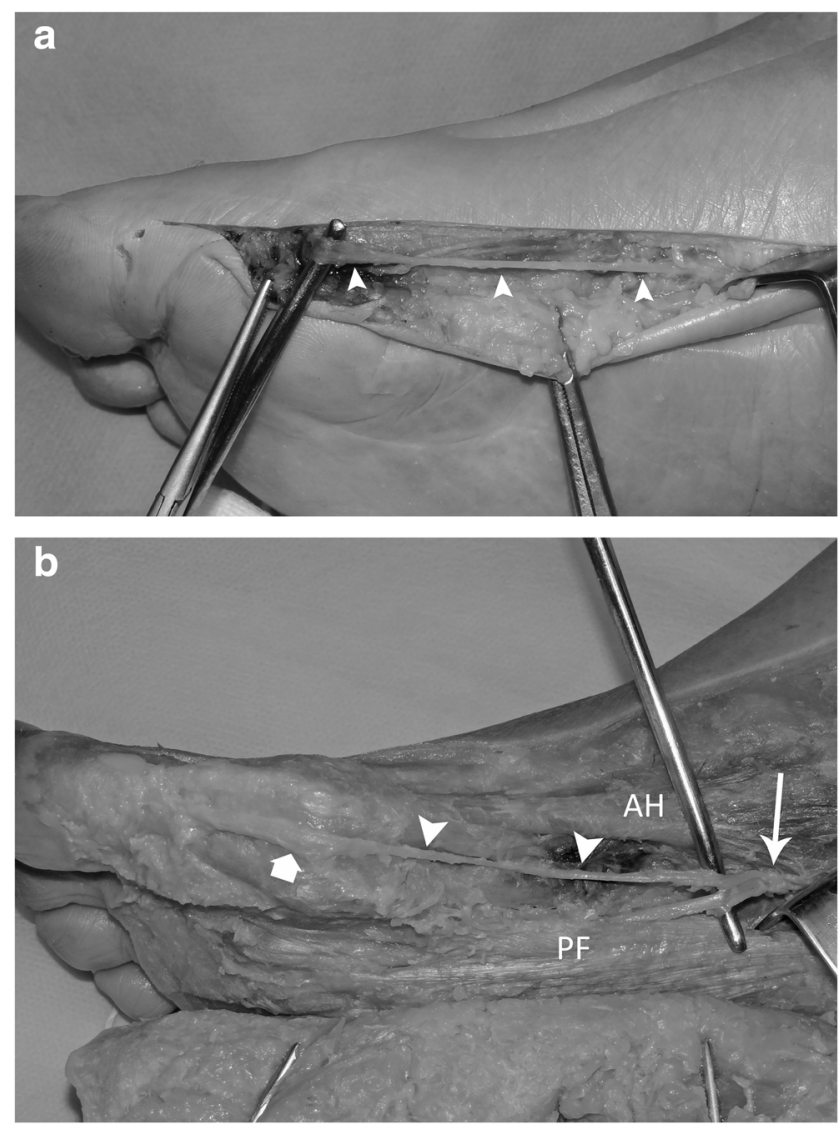

Fig. 3 Cadaver dissection of a right foot confirming that the structure visualized on US that was injected with China ink did represent the medial plantar proper digital nerve (MPPDN) (arrowheads) branching from the medial plantar nerve (MPN) (long arrow) and coursing distally between the abductor hallucis $(\mathrm{AH})$ muscle medially and plantar fascia (PF) laterally. Interestingly, the MPPDN in this specimen demonstrated a slight enlargement (thick arrow) at the level of the medial hallux sesamoid

Table 2 Results for the 17 volunteers

\begin{tabular}{ll}
\hline & Data (range) \\
\hline Mean age (y) & $32(24-43)$ \\
Mean BMI & $21.5(19.5-25)$ \\
Nerve visibility score $(0-3)$ & $2(1-3)$ \\
Cross-sectional area $\left(\mathrm{mm}^{2}\right)$ & $0.8(0.4-1.5)$ \\
D medial cuneiform $(\mathrm{mm})$ & $22(19-27)$ \\
D first metatarsal head $(\mathrm{mm})$ & $3(1-8)$ \\
D medial hallux sesamoid $(\mathrm{mm})$ & $4(2-9)$ \\
\hline
\end{tabular}

Data include demographics; the medial plantar proper digital nerve (MPPDN) visibility score ( 0 - not visible; 1 -identified with difficulty; 2-clearly identified without internal nerve structure visibility; 3 -clearly identified with internal nerve structure visibility); the MPPDN crosssectional area; and the mean distances, respectively, between the MPPDN after it branches from the medial plantar nerve and the medial border of the medial cuneiform, and at the level of the first metatarsophalangeal joint, between the nerve and the first metatarsal head, and between the nerve and the medial hallux sesamoid 
level of the base of the first metatarsal bone into two terminal branches: the medial branch that forms the first common digital nerve, and the lateral branch that forms the second and third common digital nerves [1]. In its course in the medial compartment, the MPPDN gives off a single muscular branch to the flexor hallucis brevis [3]. The nerve pierces the plantar fascia posterior to the tarsometatarsal joint and courses distally through subcutaneous tissues to supply innervation to the medial plantar aspect of the first MTPJ, hallux and tip of the toe [3-5]. Because of its particularly long course and superficial position, the MPPDN may be subject to trauma, which results in a compression neuropathy at the level of the first MTPJ known as Joplin's neuroma [6, 10]. Symptoms of Joplin's neuroma are sharp pain mixed with burning, tingling, numbness and paraesthesia to the medial and plantar aspects of the first MTPJ and hallux [3]. Any light pressure or compression, such as from a tight shoe, to the medial plantar aspect of the first MTPJ may cause severe pain and paraesthesia. The diagnosis of MPPDN neuropathy usually requires confirmation using nerve conduction studies, especially when conservative treatment is unsuccessful $[11,12]$. On the other hand, highresolution US, because it offers several advantages over other imaging modalities, has become an attractive complement for assessing the peripheral nervous system especially in the setting of a superficial neuropathy [8]. As no description of the MPPDN by means of sonography has been reported, the aim of the present study was to determine whether US allows precise assessment of the course of the MPPDN and its relations with adjacent anatomical structures.

Our results show that, despite the relative thinness of the nerve, the MPPDN could be identified sonographically along the majority of its course, from its point of branching from the medial plantar nerve below the abductor hallucis muscle to the medial side of the hallux. Here note that power Doppler imaging allowed detection of the proper plantar digital branch of the medial plantar artery adjacent to the MPPDN, which was especially helpful to identify the nerve sonographically. The MPPDN was particularly well analysed from the point where it pierces the plantar fascia to become subcutaneous to the medial plantar aspect of the first MTPJ. Here, interestingly, a slight enlargement of the nerve at the level of the medial hallux sesamoid was frequently found anatomically, which may well be a normal anatomical condition. Distally, the plantar and dorsal terminal branches of the MPPDN that supply sensation to the tip of the hallux were more difficult to assess, given their very small size. Because bony landmarks can be helpful in reliably identifying anatomical structures with US, we evaluated in both cadavers and asymptomatic volunteers the distances between the nerve and the medial border of the medial cuneiform, and, more distally, at the level of the first MTPJ, between the nerve and the first metatarsal head and the medial hallux sesamoid. In the part of our work performed on cadavers, these three distances measured by US were similar to those measured anatomically. Then, in healthy volunteers, these same three distances measured by US fell within the range demonstrated anatomically. Overall, our data suggest that the MPPDN may not only be subject to compression neuropathy at the level of the first metatarsal head, but also be at risk when performing a surgical approach to the medial plantar aspect of the first MTPJ.

Some limitations may be considered inherent to the methods used in this study. First, we dissected only six lower limbs, which is a small number for an anatomical study. Second, we did not analyse the intra- and interobserver variation in the US study of the MPPDN. The preliminary assessment of the MPPDN undertaken in cadavers and the US examinations in healthy volunteers were performed in our study by two separate teams comprised of one junior and one senior operator. Finally, the results obtained in our volunteer population might have been influenced by a 'healthy' BMI facilitating the US depiction of the nerve. It is important to note that, in patients with a higher BMI, the course of the MPPDN may be more difficult to assess with US, given the relatively small size of the nerve.

Because the anatomy of the MPPDN may vary between individuals, US mapping of the nerve may have useful clinical implications. First, because the diagnosis of Joplin's neuroma usually requires confirmation, especially when conservative

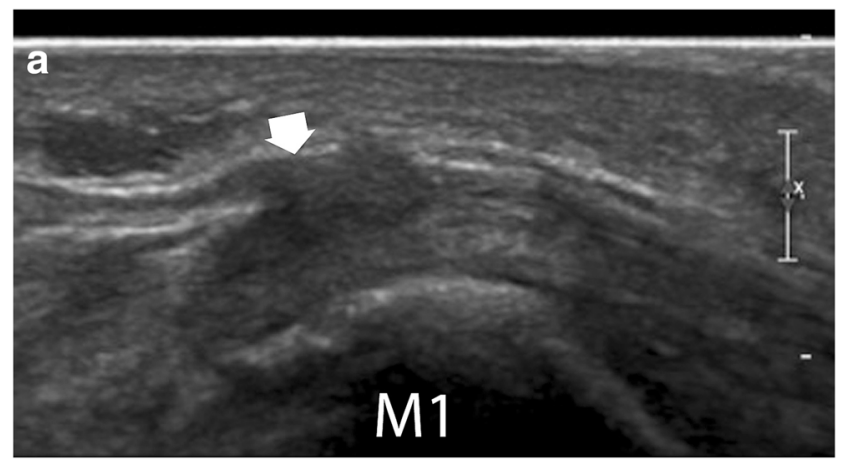

b

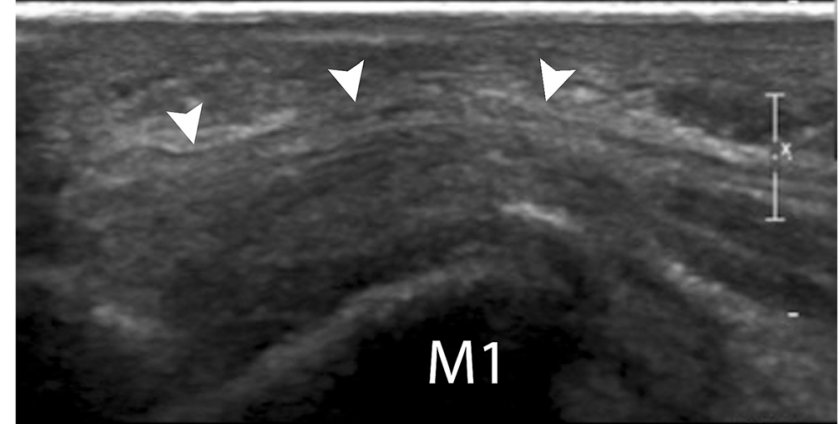

Fig. 4 Sagittal plantar bilateral US images of the first ray obtained in the same patient demonstrating a Joplin's neuroma (thick arrow), and, on the contralateral side, an intact medial plantar proper nerve (MPPN) (arrowheads) 
treatment is unsuccessful, sonography may become an attractive tool for diagnosing compression neuropathy of the MPPDN. Second, the MPPDN could be blocked successfully through US guidance, which may be a useful complement to common postoperative analgesics. Then, because injury to the MPPDN is not uncommon after bunion surgery, later resulting in neuralgia and sensory loss in its innervation area, US could help in the differential diagnosis of postoperative hallux pain (Fig. 4).

In conclusion, our study has demonstrated that, in healthy individuals, the course of the MPPDN can be depicted by US. Useful bony landmarks for its detection could be defined. The potential applications of this technique now need to be confirmed by further clinical studies.

Funding The authors state that this work has not received any funding.

\section{Compliance with ethical standards}

Guarantor The scientific guarantor of this publication is Le Corroller Thomas.

Conflict of interest The authors of this article declare no relationships with any companies whose products or services may be related to the subject matter of the article.

Statistics and biometry No complex statistical methods were necessary for this paper.

Informed consent Written informed consent was obtained from all subjects (patients) in this study.

Ethical approval Institutional Review Board approval was not required because the study only involved anatomical specimens and volunteers who were recruited from the Department of Radiology.

\section{Methodology}

- prospective

- experimental

- performed at one institution

\section{References}

1. Im S, Park JH, Kim HW, Yoo SH, Kim HS, Park GY (2010) New method to perform medial plantar proper digital nerve conduction studies. Clin Neurophysiol 121:1059-1065

2. De Maeseneer M, Madani H, Lenchik L et al (2015) Normal Anatomy and Compression Areas of Nerves of the Foot and Ankle: US and MR Imaging with Anatomic Correlation. Radiographics 35:1469-1482

3. Still GP, Fowler MB (1998) Joplin's neuroma or compression neuropathy of the plantar proper digital nerve to the hallux: clinicopathologic study of three cases. J Foot Ankle Surg 37:524-530

4. Merritt GN, Subotnick SI (1982) Medial plantar digital proper nerve syndrome (Joplin's neuroma) - typical presentation. J Foot Surg 21:166-169

5. Ames PA, Lenet MD, Sherman M (1980) Joplin's neuroma. J Am Podiatry Assoc 70:99-101

6. Joplin RJ (1971) The proper digital nerve, vitallium stem arthroplasty, and some thoughts about foot surgery in general. Clin Orthop Relat Res 76:199-212

7. Cichy SW, Claussen GC, Oh SJ (1995) Electrophysiological studies in Joplin's neuroma. Muscle Nerve 18:671-672

8. Walker FO, Cartwright MS, Wiesler ER, Caress J (2004) Ultrasound of nerve and muscle. Clin Neurophysiol 115:495-507

9. Le Corroller T, Bauones S, Acid S, Champsaur P (2013) Anatomical study of the dorsal cutaneous branch of the ulnar nerve using ultrasound. Eur Radiol 23:2246-2251

10. Jr MW, Barreira AA (1996) Joplin's neuroma. Muscle Nerve 19: 1361-1362

11. Seok HY, Eun MY, Yang HW, Lee HJ (2013) Medial plantar proper digital neuropathy caused by a ganglion cyst. Am J Phys Med Rehabil 92:1119

12. Melendez MM, Patel A, Dellon AL (2016) The Diagnosis and Treatment of Joplin's Neuroma. J Foot Ankle Surg 55:320-323 